\title{
Identification of viscoelastic properties by means of nanoindentation taking the real tip geometry into account
}

\author{
Andreas Jäger • Roman Lackner • \\ Josef Eberhardsteiner
}

Received: 24 July 2006 / Accepted: 13 November 2006 / Published online: 2 May 2007

(C) Springer Science+Business Media B.V. 2007

\begin{abstract}
Motivated by recent progress in viscoelastic indentation analysis, the identification of viscoelastic properties from nanoindentation test data taking the real tip geometry into account is presented in this paper. Based on the elastic solution of the indentation problem, the corresponding viscoelastic solution is obtained by the application of the method of functional equations. This general solution, which accounts for the real geometric properties of the indenter tip, is specialized for the case of a trapezoidal load history, commonly employed in nanoindentation testing. Three deviatoric creep models, the single dash-pot, the Maxwell, and the three-parameter model are considered. The so-obtained expressions allow us to determine viscoelastic model parameters via back calculation from the measured load-penetration history. The presented approach is illustrated by the identification of short-term viscoelastic properties of bitumen. Hereby, the influence of load-
\end{abstract}

A. Jäger $(\varangle) \cdot$ J. Eberhardsteiner Institute for Mechanics of Materials and Structures, Vienna University of Technology, Karlsplatz 13/202, 1040 Vienna, Austria

e-mail: Andreas.Jaeger@tuwien.ac.at

\section{R. Lackner}

Computational Mechanics, Technical University of

Munich, Arcisstrasse 21, 80333 Munich, Germany

e-mail: Lackner@bv.tum.de

J. Eberhardsteiner

e-mail: Josef.Eberhardsteiner@tuwien.ac.at ing rate, maximum load, and temperature on the model parameters is investigated.

Keywords Nanoindentation · Creep · Bitumen . Viscoelasticity $\cdot$ Mechanics of materials

\section{Introduction}

The main goal of nanoindentation (NI) is the identification of mechanical properties of the indented material. During NI measurements, a tip with defined shape penetrates the specimen surface with the indentation load $P[\mathrm{~N}]$ and the penetration $h[\mathrm{~m}]$ recorded as a function of time. Commonly, each indent consists of a loading, holding, and unloading phase (see Fig. 1). The hardness of the material, defined as $H=P_{\max } / A_{c}[\mathrm{~Pa}]$, is obtained from the loading phase of the NI test. Hereby, $A_{c}\left[\mathrm{~m}^{2}\right]$ is the horizontal projection of the contact area and $P_{\max }[\mathrm{N}]$ denotes the applied maximum load. According to [1,2], Young's modulus $E$ of materials exhibiting elastic or elastoplastic behavior is obtained from the relation between the measured initial slope of the unloading curve $S=\mathrm{d} P /\left.\mathrm{d} h\right|_{h=h_{\max }}$ and the indentation modulus $M=E /\left(1-v^{2}\right)$, reading

$S=\frac{2}{\sqrt{\pi}} M \sqrt{A_{c}}$

where $v$ is the Poisson's ratio. 
(a)

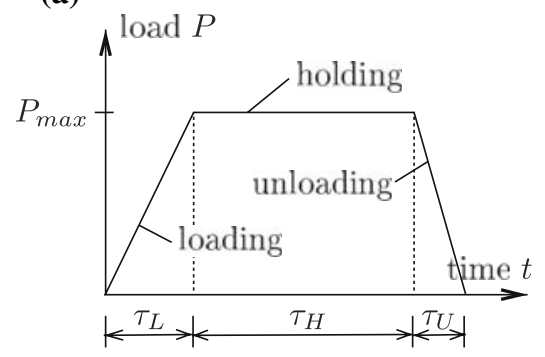

Fig. 1 Illustration of (a) load history and (b)

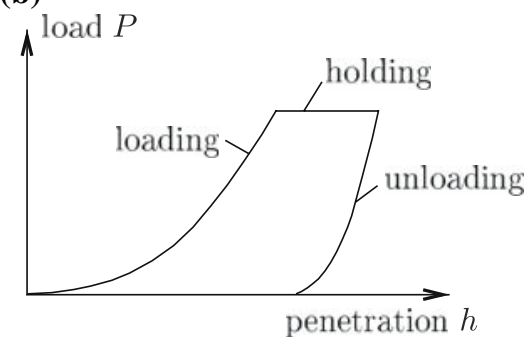

(b) load-penetration curve of NI tests
Parameter identification of materials exhibiting, in addition to elastic and plastic material response, time-dependent behavior (e.g., polymers, bitumen, etc.) requires back calculation of the parameters from the holding phase of the measured penetration history $h(t)$. Recently, analytical solutions for the indentation of axisymmetric, rigid tips into a viscoelastic halfspace were reported in [3] for spherical tips and in [4] for perfect conical tips.

Whereas both $[3,4]$ considered indenter tips characterized by exact geometric properties, the shape of real indenter tips varies in consequence of the production process and in the course of testing due to attrition. By means of calibration, NI-testing equipment give access to the real tip geometry [2]. In order to consider the so-obtained geometrical properties of the tip for back-calculation of material parameters, analytical solutions for the indentation of a tip into a viscoelastic material, taking the real tip geometry into account, are presented in this paper. For this purpose, the geometrical representation of the indenter tip (with $A_{\text {tip }}=C_{0} f(\rho)^{2}$ for perfect conical tips) is extended to

$A_{\text {tip }}(\rho)=\rho^{2} \pi=C_{0} f(\rho)^{2}+C_{1} f(\rho)$,

where $A_{\text {tip }}\left[\mathrm{m}^{2}\right]$ is the area of the cross section and $\rho[\mathrm{m}]$ and $f(\rho)[\mathrm{m}]$ are the corresponding radius and distance from the apex of the axisymmetric tip, respectively (see Fig. 2). $C_{0}[-]$ and $C_{1}[\mathrm{~m}]$ are constants describing the tip shape, which are generally provided during calibration of the NI-testing equipment. In a first step, we will solve the elastic indentation problem for the indenter shape given in Eq. 2. According to [5], the viscoelastic solution is obtained by replacing the operators of the elastic solution by the Laplace transforms of the associated viscoelastic operators. Back transformation gives access to the solution for viscoelastic inden-

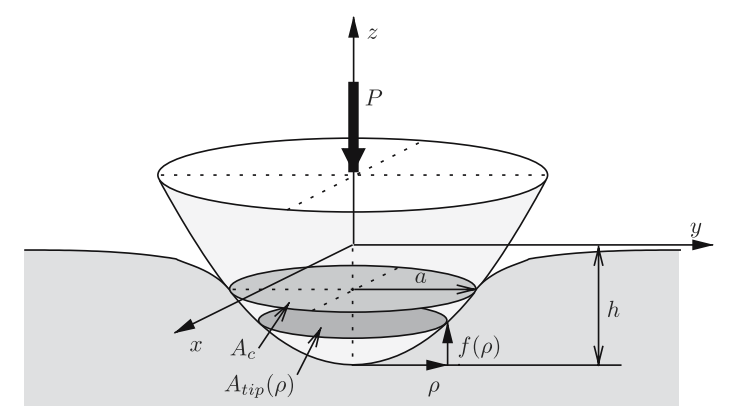

Fig. 2 Contact between a rigid axisymmetric tip of shape $f(\rho)$ and an infinite halfspace ( $P$ is the applied load, $h$ is the penetration, $a$ is the contact radius, and $A_{c}$ is the projected area of contact)

tation in the time domain. Finally, the viscoelastic solutions are employed for the identification of viscoelastic properties of bitumen from NI-test data.

\section{Elastic indentation problem}

For the solution of the elastic indentation problem, i.e., a rigid indenter penetrating the elastic halfspace, the so-called Sneddon solution [1] is employed. According to [1], the relation between the penetration $h[\mathrm{~m}]$ and the corresponding load $P$ $[\mathrm{N}]$ is given for an axisymmetric indenter tip of shape $f(\rho)$ (see Fig. 2) by

$$
\begin{aligned}
& h=a \int_{\rho=0}^{a} \frac{f^{\prime}(\rho) \mathrm{d} \rho}{\sqrt{a^{2}-\rho^{2}}} \\
& P=2 \frac{E}{1-v^{2}} \int_{\rho=0}^{a} \frac{\rho^{2} f^{\prime}(\rho) \mathrm{d} \rho}{\sqrt{a^{2}-\rho^{2}}} .
\end{aligned}
$$

Hereby, $a[\mathrm{~m}]$ is the radius of the projected contact area $A_{c}, \rho[\mathrm{m}]$ is the radius of the axisymmetric tip, $f(\rho)[\mathrm{m}]$ is a smooth function describing the tip shape, and $f^{\prime}=\mathrm{d} f / \mathrm{d} \rho$. 
For the case of conical indenters, $f(\rho)=\rho / \tan \alpha$, where $\alpha$ is the semi-apex angle. Accordingly, for the commonly used Berkovich indenter, which may be represented by a cone of $\alpha=70.32^{\circ}, f(\rho)$ becomes linear in $\rho$. In general, however, because of inaccuracies during the tip-production process and attrition, the aforementioned linear relation is nonlinear. During calibration of the NI-testing equipment, this nonlinearity is specified, following the procedure outlined in [2]:

1. Perform indents in a material with given elastic properties (e.g., fused quartz) in the depth range of the indentation experiments;

2. Compute the projected contact area $A_{c}=\pi / 4(S / M)^{2}$, where $S[\mathrm{~N} / \mathrm{m}]$ is the initial unloading slope of the load-penetration curve and $M$ is the indentation modulus, with $M=E /\left(1-v^{2}\right)$, where $E=72 \mathrm{GPa}$ and $v=0.17$ for fused quartz;

3. Plot $A_{c}$ as a function of the contact depth $h_{c}$, with $h_{c}=h-0.75 P / S$, and approximate the so-obtained function by

$A_{c}=C_{0} h_{c}^{2}+C_{1} h_{c}$

where $C_{0}[-]$ and $C_{1}[\mathrm{~m}]$ are constants describing the tip shape.

Replacing $A_{c}$ and $h_{c}$ in Eq. 4 by $\rho^{2} \pi$ and $f(\rho)$, respectively, $f(\rho)$ is obtained as

$f(\rho)=\frac{1}{2 C_{0}}\left(\sqrt{C_{1}^{2}+4 C_{0} \rho^{2} \pi}-C_{1}\right)$.

For the case of a conical indenter with a semi-apex angle $\alpha$, where $C_{0}=\pi \tan ^{2} \alpha$ and $C_{1}=0$, Eq. 5 gives $f(\rho)=\rho / \tan \alpha$. Figure 3 shows the tip-shape function $f(\rho)$ and the area function $A_{c}\left(h_{c}\right)$ for a perfect Berkovich tip $\left(C_{0}=24.5\right.$ and $\left.C_{1}=0\right)$ and a real Berkovich tip with a value of $C_{1}$ deviating from zero.

Inserting Eq. 5 into Eq. 3 gives the penetration and the applied load as a function of the contact radius $a$,

$$
\begin{aligned}
& h=a \sqrt{\frac{\pi}{C_{0}}} \arctan \frac{2 a \sqrt{C_{0} \pi}}{C_{1}}, \\
& P=4 \pi \frac{E}{1-v^{2}} \frac{2 a^{3}}{3 C_{1}}{ }_{2} F_{1}\left(1 / 2 ; 2 ; 5 / 2 ;-\frac{4 a^{2} C_{0} \pi}{C_{1}^{2}}\right),
\end{aligned}
$$

where ${ }_{2} F_{1}(a ; b ; c ; z)$ denotes a hypergeometric function, defined by (see, e.g., [6])

$$
\begin{aligned}
{ }_{2} F_{1}(a ; b ; c ; z)= & \frac{\Gamma(c)}{\Gamma(b) \Gamma(c-b)} \\
& \times \int_{0}^{1} \frac{t^{b-1}(1-t)^{c-b-1}}{(1-t z)^{a}} \mathrm{~d} t,
\end{aligned}
$$

which is only valid for $\operatorname{Re}(c)>\operatorname{Re}(b)>0$.

\section{Viscoelastic indentation problem - application to trapezoidal load history}

In the following, the elastic indentation problem outlined in the previous section is extended to linear viscoelasticity. For this purpose, the following two methods may be employed:

1. Laplace transform method: The Laplace transform method consists in removing the time variable in the viscoelastic problem by employing the Laplace transformation to the time-dependent equations [7]. From the solution of the so-obtained associated elastic problem, the time-dependent solution is obtained by application of the inverse Laplace transformation. However, this method is restricted to problems, where the interface between deformation and stress boundaries does not change with time, which is not the case for indentation problems.

2. Method of functional equations: In the case of the considered indentation problems, the method of functional equations, developed by [5], consists in replacing the elastic constants in the solution of the equivalent elastic boundary value problem by the Laplace transforms of the associated viscoelastic operators. This method is not restricted to fixed boundary conditions and remains valid as long as the contact area increases monotonically with time [5].

Due to the restrictions of the Laplace transform method, the method of functional equations (see also [3,4]) is employed to determine the viscoelastic solution for tip shapes described by Eq. 2 . Hereby, the result for the solution of the indentation in an elastic halfspace (Eq. 7) is rewritten in the form

$P=M F(a)$. 

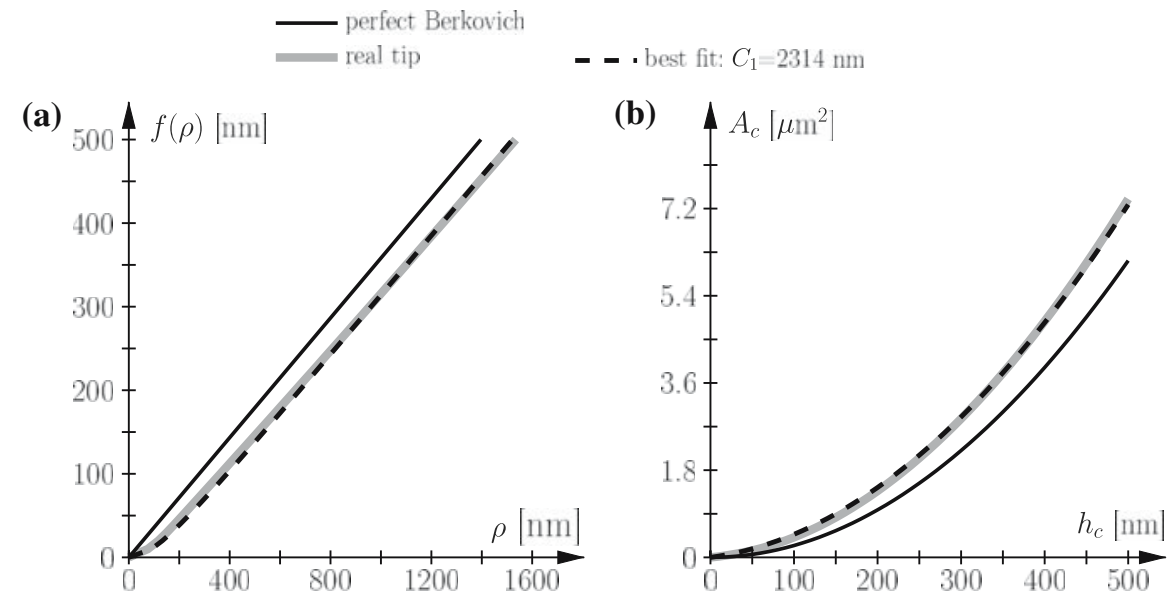

Fig. 3 (a) Tip-shape function $f(\rho)$ and (b) area function $A_{c}\left(h_{c}\right)$ of a perfect Berkovich tip $\left(C_{0}=24.5\right.$ and $\left.C_{1}=0\right)$, and a real Berkovich tip with $C_{0}=24.5$ and $C_{1}=2314 \mathrm{~nm}$

Thus, the solution is split into the material dependent indentation modulus $M$, with $M=E /\left(1-v^{2}\right)$, and the function $F(a)$ depending only on geometric properties, such as the tip shape (represented by the constant parameters $C_{0}$ and $C_{1}$ ) and the unknown contact radius $a$, reading

$F(a)=4 \pi \frac{2 a^{3}}{3 C_{1}}{ }_{2} F_{1}\left(1 / 2 ; 2 ; 5 / 2 ;-\frac{4 a^{2} C_{0} \pi}{C_{1}^{2}}\right)$.

Following the method of functional equations, the viscoelastic solution for the indentation problem is obtained by replacing the elastic operators $P$, $M$, and $F(a)$ in Eq. 10 by their Laplace transforms $\widehat{P(s)}, \widehat{M(s)}$, and $\widehat{F(a(s))}$, giving

$\widehat{P(s)}=\widehat{M(s)} \widehat{F(a(s))}$.

Re-arrangement yields an expression for the Laplace transform of the function $F(a(s))$ as

$\widehat{F(a(s)})=\frac{\widehat{P(s)}}{\widehat{M(s)}}=\frac{1}{s \widehat{M(s)}} s \widehat{P(s)}=\widehat{\widehat{Y}(s)} s \widehat{P(s)}$,

where $1 /(s \widehat{M(s)})$ was replaced by the Laplace transform of $\bar{Y}(t)$, in the following referred to as indentation compliance function. Considering that (i) a multiplication by $s$ in the Laplace domain is equivalent to a derivation in the time domain and (ii) a multiplication of two Laplace-transformed functions is equivalent to the convolution product of the two functions in the time domain, $F(a(t))$ is obtained from Eq. 12 as
$F(a(t))=\int_{0}^{t} \bar{Y}(t-\tau) \frac{\mathrm{d}}{\mathrm{d} \tau} P(\tau) \mathrm{d} \tau$.

Finally, combining Eqs. 10 and 13 allows determination of the unknown contact radius $a(t)$. With $a(t)$ at hand, Eq. 6 provides access to the history of the penetration, $h(t)$.

The method of functional equations is restricted to increasing contact areas, and may be applied only to monotonically increasing and constant load histories [5]. Since indentation tests are commonly conducted under load control, Eq. 13 is specified to the trapezoidal load history depicted in Fig. 1(a), reading

$$
P(t)=\left\{\begin{aligned}
& P_{L}(t)=t / \tau_{L} P_{\max } \text { for } 0 \leq t \leq \tau_{L}, \\
& P_{H}(t)=P_{\max } \quad \text { for } \tau_{L} \leq t \leq \tau_{L}+\tau_{H} \\
& P_{U}(t)=\frac{\tau_{L}+\tau_{H}+\tau_{U}-t}{\tau_{U}} P_{\max } \\
& \text { for } \tau_{L}+\tau_{H} \leq t \\
& \quad \leq \tau_{L}+\tau_{H}+\tau_{U},
\end{aligned}\right.
$$

where $\tau_{L}, \tau_{H}$, and $\tau_{U}$ are the loading, holding, and unloading durations, respectively. Considering the load history $P(t)$ given in Eq. 14 in Eq. 13, the function $F(a(t))$ becomes for the loading and holding regime

$$
\begin{aligned}
F_{L}(a(t)) & =\int_{0}^{t} \bar{Y}(t-\tau) \frac{\mathrm{d}}{\mathrm{d} \tau} P_{L}(\tau) \mathrm{d} \tau \\
& =\frac{P_{\max }}{\tau_{L}} \int_{0}^{t} \bar{Y}(t-\tau) \mathrm{d} \tau,
\end{aligned}
$$


(a) (b) (c)

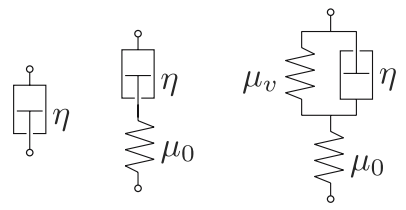

Fig. 4 Viscoelastic deviatoric creep models considered in this study: (a) the single dash-pot (DP), (b) the Maxwell (MX) model, and (c) the three-parameter (3P) model

$$
\begin{aligned}
F_{H}(a(t))= & \int_{0}^{\tau_{L}} \bar{Y}(t-\tau) \frac{\mathrm{d}}{\mathrm{d} \tau} P_{L}(\tau) \mathrm{d} \tau \\
& +\int_{\tau_{L}}^{t} \bar{Y}(t-\tau) \frac{\mathrm{d}}{\mathrm{d} \tau} P_{H}(\tau) \mathrm{d} \tau \\
= & \frac{P_{\max }}{\tau_{L}} \int_{0}^{\tau_{L}} \bar{Y}(t-\tau) \mathrm{d} \tau .
\end{aligned}
$$

Based on $F_{L}$ and $F_{H}$ in Eqs. 15 and 16, the history of the penetration, $h(t)$, for the loading and holding time is determined in three steps:

1. The indentation compliance function $\bar{Y}(t)$ appearing in Eqs. 15 and 16 is determined for the considered viscoelastic model;

2. $\quad F_{L}(a(t))$ and $F_{H}(a(t))$ are computed using Eqs. 15 and 16 ; and

3. The contact radius $a(t)$ and, hence, via Eq. 6, the penetration $h(t)$ are determined by combining the expressions for $F_{L}(a(t))$ and $F_{H}(a(t))$ with Eq. 10.

In the following subsections, these three steps are described in more detail.

3.1 Determination of the indentation compliance function $\bar{Y}(t)$

The indentation compliance function $\bar{Y}(t)$ is determined for three deviatoric creep models, i.e., (i) the single dash-pot (DP), (ii) the Maxwell (MX) model, and (iii) the three-parameter (3P) model (see Fig. 4). In the case of elastic material response, the indentation modulus $M$ can be expressed by the bulk modulus $k$ and the shear modulus $\mu_{0}$, reading

$$
\begin{aligned}
M=\frac{E}{1-v^{2}} & =\frac{9 k \mu_{0} /\left(3 k+\mu_{0}\right)}{1-\left(\left(3 k-2 \mu_{0}\right) /\left(6 k+2 \mu_{0}\right)\right)^{2}} \\
& =4 \mu_{0} \frac{3 k+\mu_{0}}{3 k+4 \mu_{0}} .
\end{aligned}
$$

By the way of application of the method of functional equations, the elastic constants $k$ and $\mu_{0}$ in Eq. 17 are replaced by the associated Laplacetransformed operators $\widehat{k(s)}$ and $\widehat{\mu(s)}$, reading

$\widehat{M(s)}=4 \widehat{\mu(s)} \frac{3 \widehat{k(s)}+\widehat{\mu(s)}}{3 \widehat{k(s)}+4 \widehat{\mu(s)}}$,

where $\widehat{k(s)}=k$ for the case of deviatoric creep only. The shear relaxation modulus, on the other hand, is time dependent, and is given by (see, e.g., [8]):

$$
\begin{aligned}
\widehat{\mu_{D P}(s)} & =\left(s \mathcal{L}\left\{J_{D P}\right\}\right)^{-1}=(s \mathcal{L}\{t / \eta\})^{-1}=s \eta \\
\widehat{\mu_{M X}(s)} & =\left(s \mathcal{L}\left\{J_{M X}\right\}\right)^{-1}=\left(s \mathcal{L}\left\{1 / \mu_{0}+t / \eta\right\}\right)^{-1} \\
& =\left(\frac{1}{\mu_{0}}+\frac{1}{s \eta}\right)^{-1} \\
\widehat{\mu_{3 P}(s)} & =\left(s \mathcal{L}\left\{J_{3 P}\right\}\right)^{-1} \\
& =\left(s \mathcal{L}\left\{\frac{1}{\mu_{0}}+\frac{1}{\mu_{v}}\left[1-\exp \left(-\frac{\mu_{v}}{\eta} t\right)\right]\right\}\right)^{-1} \\
& =\left(\frac{1}{\mu_{0}}+\frac{1}{\mu_{v}+s \eta}\right)^{-1}
\end{aligned}
$$

where $\mathcal{L}\{\bullet(t)\}$ denotes the Laplace transformation of $\bullet(t)$. Considering $\widehat{\mu(s)}$ of the different viscoelastic models given in Eqs. 19-21 in Eq. 18 andapplying the inverse Laplace transformation to $\widehat{\bar{Y}(s)}=1 /(s \widehat{M(s)}), \bar{Y}(t)$ is obtained as

$$
\begin{aligned}
\bar{Y}_{D P}(t)= & \frac{1}{4}\left\{\frac{t}{\eta}+\frac{1}{k}\left[1-\exp \left(-\frac{3 k}{\eta} t\right)\right]\right\}, \\
\bar{Y}_{M X}(t)= & \frac{1}{4}\left\{\frac{1}{\mu_{0}}+\frac{t}{\eta}+\frac{1}{k}\left[1-\frac{\mu_{0}}{\mu_{0}+3 k}\right.\right. \\
& \left.\left.\exp \left(-\frac{3 k \mu_{0}}{\eta\left(\mu_{0}+3 k\right)} t\right)\right]\right\}, \\
\bar{Y}_{3 P}(t)= & \frac{1}{4}\left\{\frac{1}{\mu_{0}}+\frac{1}{\mu_{v}}\left[1-\exp \left(-\frac{\mu_{v}}{\eta} t\right)\right]\right. \\
& +\frac{3\left(\mu_{0}+\mu_{v}\right)}{\mu_{0} \mu_{v}+3 k\left(\mu_{0}+\mu_{v}\right)} \\
& \times\left[1-\frac{\mu_{0}^{2}}{\left(\mu_{0}+3 k\right)\left(\mu_{0}+\mu_{v}\right)}\right. \\
& \left.\left.\exp \left(-\frac{\mu_{0} \mu_{v}+3 k\left(\mu_{0}+\mu_{v}\right)}{\eta\left(\mu_{0}+3 k\right)} t\right)\right]\right\} .
\end{aligned}
$$


In the case of incompressible materials, where $k=$ $\infty$, Eqs. 22-24 simplify to

$\bar{Y}_{D P}(t)=\frac{1}{4} \frac{t}{\eta}$,

$\bar{Y}_{M X}(t)=\frac{1}{4}\left(\frac{1}{\mu_{0}}+\frac{t}{\eta}\right)$,

$\bar{Y}_{3 P}(t)=\frac{1}{4}\left\{\frac{1}{\mu_{0}}+\frac{1}{\mu_{v}}\left[1-\exp \left(-\frac{\mu_{v}}{\eta} t\right)\right]\right\}$.

\subsection{Determination of $F(a(t))$}

Considering the indentation compliance functions $\bar{Y}(t)$ for the three viscoelastic models given in Eqs. 22-24 in Eqs. 15 and 16, the function $F(a(t))$ is obtained for the loading and holding regime, $F_{L}(a(t))$ and $F_{H}(a(t))$, for the three considered viscoelastic models as

\subsection{Determination of $a(t)$ and $h(t)$}

The history of the contact radius, $a(t)$, is obtained from combining the expressions for $F_{L}(a(t))$ and $F_{H}(a(t))$ given in Eqs. 28-33 with Eq. 10. The so-obtained (nonlinear) expression for $a(t)$ is solved numerically, employing a Newton-iteration scheme. With $a(t)$ at hand, the history of the penetration, $h(t)$, is given by Eq. 6 for a given load history $P(t)$ and the material model describing the behavior of the viscoelastic half space.

\subsection{Illustrative example}

The developed mode of determination of the penetration history $h(t)$ provides insight into the influence of the tip shape on NI results, as illustrated for a DP-type material in Fig. 5. According

$$
\begin{aligned}
& F_{L-D P}(a(t))=\frac{P_{\max }}{\tau_{L}} \int_{0}^{t} \bar{Y}(t-\tau) \mathrm{d} \tau \\
& =\frac{P_{\max }}{4 \tau_{L}}\left\{\frac{1}{k} t+\frac{1}{2 \eta} t^{2}-\frac{\eta}{3 k^{2}}\left(1-\exp \left(-\frac{3 k}{\eta} t\right)\right)\right\}, \\
& F_{H-D P}(a(t))=\frac{P_{\max }}{\tau_{L}} \int_{0}^{\tau_{L}} \bar{Y}(t-\tau) \mathrm{d} \tau \\
& =\frac{P_{\max }}{4 \tau_{L}}\left\{\frac{\tau_{L}}{k}+\frac{1}{2 \eta}\left(2 t-\tau_{L}\right) \tau_{L}+\frac{\eta}{3 k^{2}}\left(\exp \left(-\frac{3 k}{\eta} t\right)\left[1-\exp \left(\frac{3 k}{\eta} \tau_{L}\right)\right]\right)\right\}, \\
& F_{L-M X}(a(t))=\frac{P_{\max }}{4 \tau_{L}}\left\{\frac{\mu_{0}+k}{\mu_{0} k} t+\frac{1}{2 \eta} t^{2}-\frac{\eta}{3 k^{2}}\left(1-\exp \left(-\frac{3 \mu_{0} k}{\eta\left(\mu_{0}+3 k\right)} t\right)\right)\right\}, \\
& F_{H-M X}(a(t))=\frac{P_{\max }}{4 \tau_{L}}\left\{\frac{\mu_{0}+k}{\mu_{0} k} \tau_{L}+\frac{1}{2 \eta}\left(2 t-\tau_{L}\right) \tau_{L}+\frac{\eta}{3 k^{2}}\left(\exp \left(-\frac{3 \mu_{0} k}{\eta\left(\mu_{0}+3 k\right)} t\right)\right.\right. \\
& \left.\left.\times\left[1-\exp \left(\frac{3 \mu_{0} k}{\eta\left(\mu_{0}+3 k\right)} \tau_{L}\right)\right]\right)\right\}, \\
& F_{L-3 P}(a(t))=\frac{P_{\max }}{4 \tau_{L}}\left\{\frac{3 \mu_{0}^{2} \eta}{\left(\mu_{0} \mu_{v}+3 k\left(\mu_{0}+\mu_{v}\right)\right)^{2}}\left(-1+\exp \left(-\frac{\mu_{0} \mu_{v}+3 k\left(\mu_{0}+\mu_{v}\right)}{\eta\left(\mu_{0}+3 k\right)} t\right)\right)\right. \\
& \left.+\frac{\left(\mu_{0}+\mu_{v}\right)\left(4 \mu_{0} \mu_{v}+3 k\left(\mu_{0}+\mu_{v}\right)\right)}{\mu_{0} \mu_{v}\left(\mu_{0} \mu_{v}+3 k\left(\mu_{0}+\mu_{v}\right)\right)} t+\frac{\eta}{\mu_{v}^{2}}\left(-1+\exp \left(-\frac{\mu_{v}}{\eta} t\right)\right)\right\}, \\
& F_{H-3 P}(a(t))=\frac{P_{\max }}{4 \tau_{L}}\left\{\left(\frac{1}{\mu_{0}}+\frac{1}{\mu_{v}}+\frac{3\left(\mu_{0}+\mu_{v}\right)}{\mu_{0} \mu_{v}+3 k\left(\mu_{0}+\mu_{v}\right)}\right) \tau_{L}-\frac{\eta}{\mu_{v}^{2}}\left(\exp \left(-\frac{\mu_{v}}{\eta} t\right)\left[-1+\exp \left(\frac{\mu_{v}}{\eta} \tau_{L}\right)\right]\right)\right. \\
& -\frac{3 \eta \mu_{0}^{2}}{\left(\mu_{0} \mu_{v}+3 k\left(\mu_{0}+\mu_{v}\right)\right)^{2}}\left(\exp \left(-\frac{\mu_{0} \mu_{v}+3 k\left(\mu_{0}+\mu_{v}\right)}{\eta\left(\mu_{0}+3 k\right)} t\right)\right. \\
& \left.\left.\times\left[-1+\exp \left(\frac{\mu_{0} \mu_{v}+3 k\left(\mu_{0}+\mu_{v}\right)}{\eta\left(\mu_{0}+3 k\right)} \tau_{L}\right)\right]\right)\right\} \text {. }
\end{aligned}
$$


to Fig. 5(a), the largest penetration is obtained for the perfect Berkovich. Any variation of the indenter tip from the perfect Berkovich tip results in a reduction of the penetration. Figure 5(b) shows the deviation of the penetration depth from the Berkovich response, giving deviations found in the tens of percent.

\section{Application -identification of viscoelastic properties of bitumen}

\subsection{Introductionary remarks}

Bitumen is the binder material of asphalt and determines its complex thermo-rheological behavior which, at high temperatures $\left(T>135^{\circ} \mathrm{C}\right)$, provides the low viscosity of asphalt required for the construction and compaction process of high-quality asphalt layers. During hot summer periods, this viscosity should be significantly higher in order to avoid the development of permanent deformations in asphalt pavements (rutting), requiring costly repair work and reducing the traffic safety. The desirable increase of viscosity from hot to medium temperatures $\left(0<T<70^{\circ} \mathrm{C}\right)$ is, on the other hand, disadvantageous at low-temperatures $\left(T<0^{\circ} \mathrm{C}\right)$, causing low-temperature cracking in asphalt pavements in consequence of thermalshrinkage strains associated with cooling during changing weather conditions.

In general, the viscosity of bitumen is found to decrease linearly with increasing temperature in the $\log$ (viscosity)-temperature diagram (see Fig. 6 [9]). The viscosity and its change with increasing temperature is influenced by several factors:

1. The chemical composition and the molecular weight distribution of the crude oil;

2. The so-called cut point, i.e., the temperature during the distillation process (the higher the cut point, the higher the viscosity), and air blowing after the distillation process (increase of viscosity due to bitumen oxidation); and

3. The allowance of additives (commonly polymers).

In order to gain insight in the origin of the bitumen viscosity, the viscous properties of one chosen type of bitumen (B50/70, see Table 1) are determined,
Table 1 Properties of considered type of bitumen (B50/70)

\begin{tabular}{ll}
\hline Penetration depth $[1 / 10 \mathrm{~mm}][10]$ & 49 \\
Breaking point by $\mathrm{Fraß}\left[{ }^{\circ} \mathrm{C}\right][11]$ & -13 \\
Softening point $\left[{ }^{\circ} \mathrm{C}\right][12]$ & 50.5 \\
$\quad$ Elemental analysis [mass-\%] & \\
Carbon & 83.04 \\
Hydrogen & 10.38 \\
Nitrogen & 1.11 \\
Sulfur & 5.05 \\
$\Sigma$ & 99.58 \\
\hline
\end{tabular}

employing the parameter-identification procedure outlined in the previous section.

\subsection{Specimen preparation and test protocol}

In addition to the identification of model parameters, the application of the so-called grid indentation technique [13] gives access to the morphology of bitumen in the $\mu \mathrm{m}$ range and allows application of statistical techniques for the interpretation of NI-test results. Hereby, several indents are performed on a specified grid (e.g., $10 \times 10$ indents). The distance between two adjacent grid points is adjusted to the characteristic dimension of the bitumen microstructure and the maximum penetration.

The bitumen samples were prepared by heating and pouring into a sample holder. The bitumen surface obtained from pouring (as opposed to low-temperature fracture) exhibits the appropriate smoothness for NI testing. Two studies were performed, focusing on

- The influence of the loading rate and the maximum load on the determined model parameters and

- The temperature dependence of the model parameters.

\subsection{Presentation of results and discussion}

In order to determine model parameters for bitumen from NI data, the analytical solutions for $F_{L}(a(t))$ and $F_{H}(a(t))$ (see Eqs. 28-33) are specialized for incompressible materials, i.e., for $k=\infty$, giving

$$
F_{L-D P}(a(t))=\frac{P_{\max }}{8 \tau_{L} \eta} t^{2},
$$



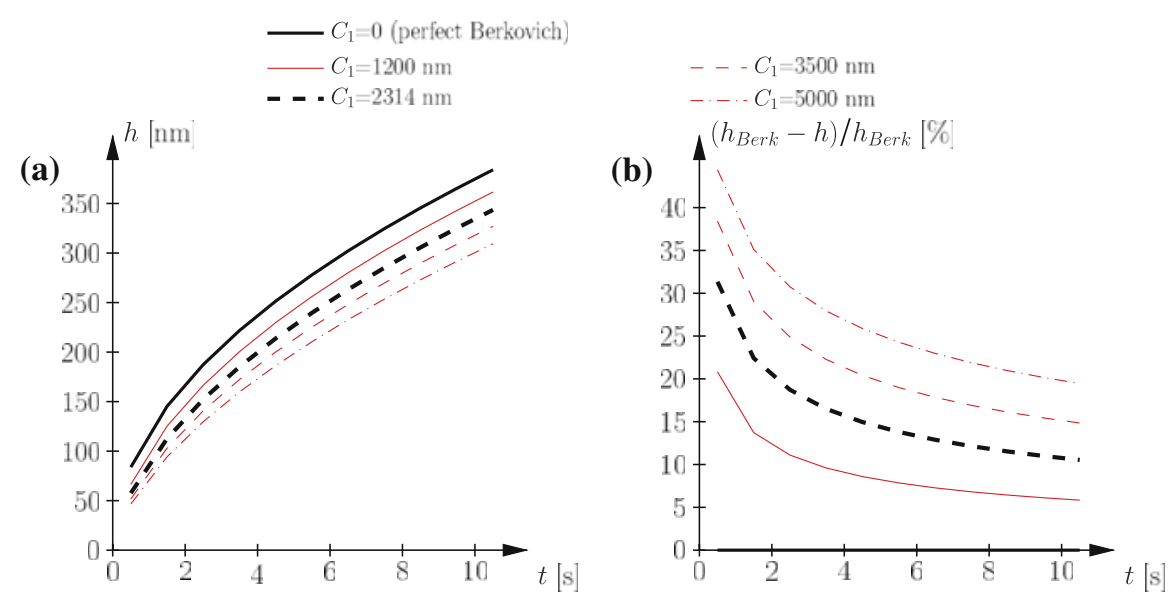

Fig. 5 Indentation of viscoelastic halfspace (DP-model with $\eta=100 \mathrm{MPa}$ s) for different tip shapes: (a) Penetration history of the holding period; (b) deviation of penetration depth from response obtained from perfect Berkovich indenter $\left(C_{0}=24.5\right.$; load history: $P_{\max }=10 \mu \mathrm{N}, \tau_{L}=0.5 \mathrm{~s}, \tau_{H}=10 \mathrm{~s}$ )

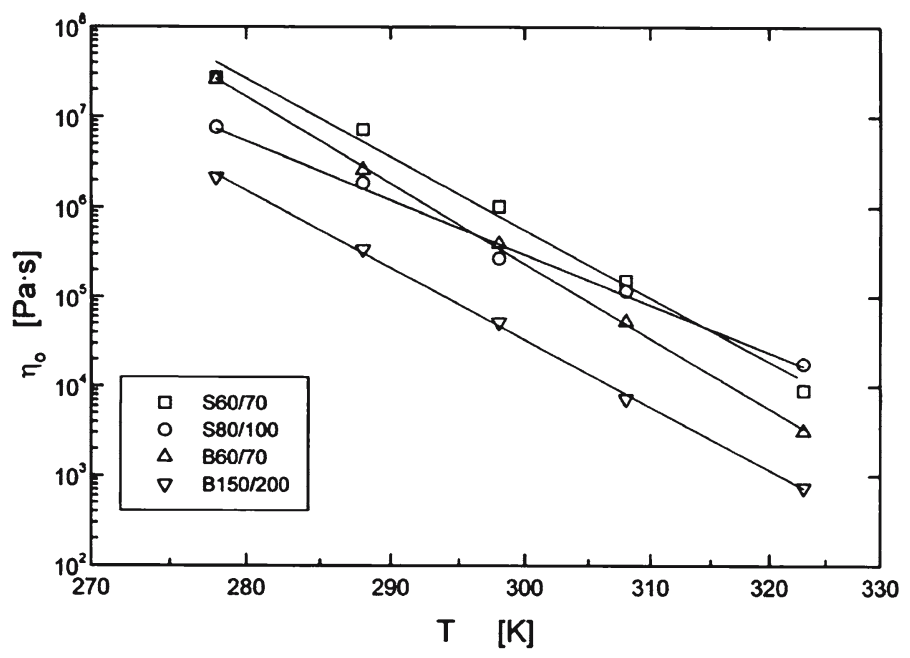

Fig. 6 Temperature dependence of the zero-shear-rate limiting viscosity for different types of bitumen [9]

$$
\begin{aligned}
F_{H-D P}(a(t))= & \frac{P_{\max }}{8 \eta}\left(2 t-\tau_{L}\right), \\
F_{L-M X}(a(t))= & \frac{P_{\max }}{4 \tau_{L}}\left\{\frac{1}{\mu_{0}} t+\frac{1}{2 \eta} t^{2}\right\}, \\
F_{H-M X}(a(t))= & \frac{P_{\max }}{4}\left\{\frac{1}{\mu_{0}}+\frac{1}{2 \eta}\left(2 t-\tau_{L}\right)\right\}, \\
F_{L-3 P}(a(t))= & \frac{P_{\max }}{4 \tau_{L}}\left\{\left(\frac{1}{\mu_{0}}+\frac{1}{\mu_{v}}\right) t\right. \\
& \left.+\frac{\eta}{\mu_{v}^{2}}\left[-1+\exp \left(-\frac{\mu_{v}}{\eta} t\right)\right]\right\},
\end{aligned}
$$

$$
\begin{aligned}
F_{H-3 P}(a(t))= & \frac{P_{\max }}{4 \tau_{L}}\left\{\left(\frac{1}{\mu_{0}}+\frac{1}{\mu_{v}}\right) \tau_{L}-\frac{\eta}{\mu_{v}^{2}}\right. \\
& \left.\exp \left(-\frac{\mu_{v}}{\eta} t\right)\left[-1+\exp \left(\frac{\mu_{v}}{\eta} \tau_{L}\right)\right]\right\} .
\end{aligned}
$$

Taking into account that the MX and the DP model are special cases of the more general 3P model, giving the MX model for $\mu_{v}=0$ and the DP model for $\mu_{v}=0, \mu_{0}=\infty$, the $3 \mathrm{P}$ model is employed in the following for the identification of model parameters. Hereby, the error between the experimentally 

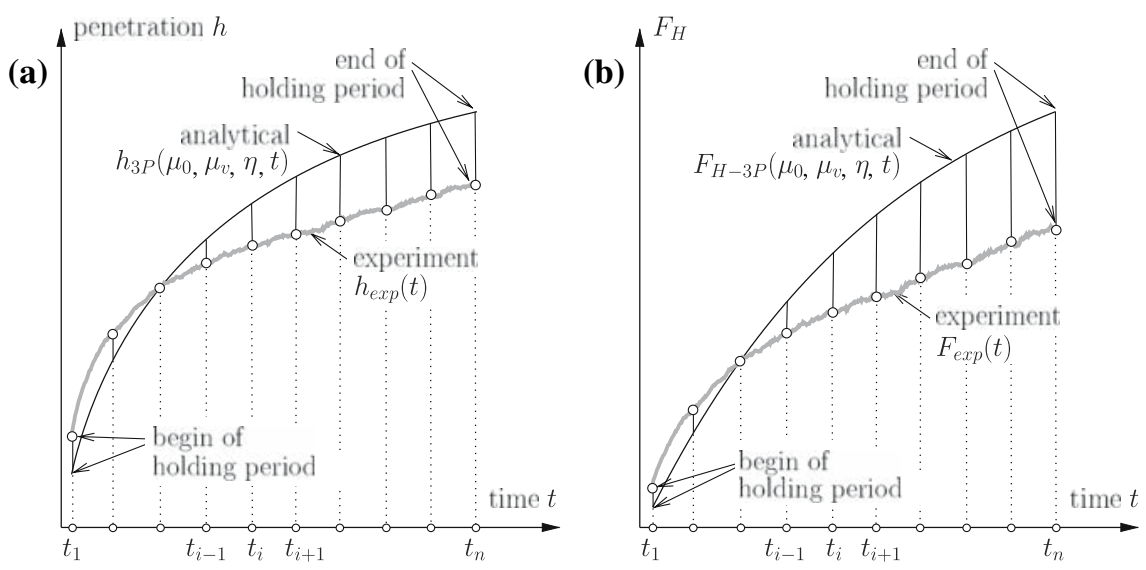

Fig. 7 Illustration of error between model response and NI-test data (holding period): (a) penetration history $h(t)$ and (b) function $F_{H}$

obtained function $F_{\exp }(a(t))^{1}$ for the holding period and the analytical result given in Eq. (39) is minimized by adapting the unknown shear moduli $\mu_{0}$ and $\mu_{v}$, and the viscosity $\eta$ (see Fig. 7). The mentioned error is defined by

$R_{3 P}\left(\mu_{0}, \mu_{v}, \eta\right)=\frac{e\left(\mu_{0}, \mu_{v}, \eta\right)}{u}$

with

$$
\begin{aligned}
e^{2}\left(\mu_{0}, \mu_{v}, \eta\right)= & \sum_{i=1}^{n}\left[F_{\exp }\left(t_{i}\right)-F_{H-3 P}\left(\mu_{0}, \mu_{v}, \eta, t_{i}\right)\right]^{2} \\
& \text { and } u^{2}=\sum_{i=1}^{n} F_{\exp }^{2}\left(t_{i}\right),
\end{aligned}
$$

where $n$ was set equal to 10 . The error given in Eq. 40 was minimized by adapting $\mu_{0}, \mu_{v}$, and $\eta$, using a simplex iteration [14]. With the model parameters at hand, the history of the contact radius, $a_{3 P}\left(t_{i}\right)$, is determined from Eq. 10 using a Newton-iteration scheme. Subsequently, $h_{3 P}\left(t_{i}\right)$ is computed from Eq. 6 (see, e.g, Fig. 7(a)). The procedure employed for parameter identification is summarized in Fig. 8.

In order to check the existence of a global minimum of the error $R_{3 P}$, the influence of the model parameters on the error $R_{3 P}$ is studied. Figure 9 shows contour-plots of the error as a function of

$\overline{1 F_{\text {exp }}(a(t))}$ is determined from the penetration history using Eqs. 6 and 10 and $C_{0}=24.5 ; C_{1}$ is adapted to the penetration at the end of the holding period using the NI-equipment calibration data. the model parameters (one parameter is kept constant for each plot). Whereas the spring in series $\left(\mu_{0}\right)$ only slightly changes the error, variation of the parameters of the Kelvin-Voigt unit ( $\eta$ and $\left.\mu_{v}\right)$ significantly affects the error. The distribution of error $R_{3 P}$ shown in Fig. 9 indicates the existence of one global minimum.

The presented parameter-identification scheme was applied to the considered type of bitumen tested at $-1^{\circ} \mathrm{C}$. Hereby, 100 indents in a $10 \times 10$ grid were performed. Figure 10 shows the frequency plots for the Young's modulus $E_{0}$, with $E_{0}=3 \mu_{0}$ using Poisson's ratio $v=0.5$ for incompressible materials, the shear modulus $\mu_{v}$, the viscosity $\eta$, and the error $R_{3 P}$. The computed mean value of the error $R_{3 P}$ does not exceed $4 \%$, confirming the proper choice of the $3 \mathrm{P}$ model for fitting the short-term viscoelastic response of bitumen. The obtained frequency plots are approximated by a Gaussian distribution, giving mean values and standard deviations for the model parameters. For the considered type of bitumen (B50/70) the mean values of the model parameters at $T=-1{ }^{\circ} \mathrm{C}$ are: $E_{0}=3 \mu_{0}=2.0 \mathrm{GPa}, \mu_{v}=0.022 \mathrm{GPa}$, and $\eta=0.15 \mathrm{GPa}$.

Figure 11 shows the mean values of the 3Pmodel parameters for different loading rates and different values for the maximum load identified from grid indentations on bitumen B50/70 at $-1^{\circ} \mathrm{C}(\dot{P}=\mathrm{d} P / \mathrm{d} t=20,40,80,160 \mu \mathrm{N} / \mathrm{s}$; $\left.P_{\max }=10,20,50,120,240 \mu \mathrm{N}\right)$. Whereas the influence of the loading rate on the parameters 
1. Obtain maximum penetration, $h_{\max }$, from indentation data

2. Compute $C_{1}$ for $h_{\max }$ according to Equation (4) using a Newton-iteration scheme $\left(C_{0}=24.5, A_{c}\left(\mathrm{~h}_{c}\right)\right.$ is provided from the NI-equipment calibration)

3. Select $n$ equidistant data points within the holding period for error minimization

4. DO $i=1, n$
a) Compute contact radius $a_{\text {exp }}\left(t_{i}\right)$ from Equation (6) using a Newton-iteration scheme
b) Compute $F_{\text {exp }}\left(a\left(t_{i}\right)\right)$ from Equation (10)
ENDDO

5. Identify model parameters $\mu_{0}, \mu_{v}$, and $\eta$ using a simplex iteration [14]

a) Determine start values based on analytical results for perfect conical indentation [4]

b) Minimize error $R_{3 P}$ given in Equation (40)

The penetration history corresponding to the identified model parameters may be obtained from Equations (39), $(10)$, and (6), giving $F_{H-3 P}(t), a_{3 P}(t)$, and finally $h_{3 P}(t)$.

Fig. 8 Flowchart for identification of model parameters for one indent

is small for all considered load levels, the maximum load itself has a significant impact on the obtained model parameters. By increasing the maximum load, the model parameters decrease until they reach a limiting value. The error $R_{3 P}$ (Eq. 40), reflecting the deviation between NI-test data and model response, is always smaller than 4\%. Accordingly, an extension of the employed 3P-model would, of course, further reduce the aforementioned deviation, but would not eliminate the observed load-dependency of the identified model parameters. Thus, the variation of the identified model parameters with the maximum load and, consequently, with the penetration depth is explained by the bitumen microstructure, consisting of high-viscous strings embedded into a low-viscous matrix [15].

Accounting for the thermo-rheological behavior of bitumen, the influence of temperature on the model parameters is studied by conducting NI tests at different temperatures. Figures 12(a), (b), and (c) show mean values of the 3P-model parameters for bitumen B50/70 tested at $T=-4.5,-1,2$, 5.5 , and $9^{\circ} \mathrm{C}$. All three parameters exhibit the same temperature dependence, decreasing from $-4.5^{\circ} \mathrm{C}$ to $-1^{\circ} \mathrm{C}$, slightly increasing from $-1^{\circ} \mathrm{C}$ to $2^{\circ} \mathrm{C}$, and finally decreasing again from $2{ }^{\circ} \mathrm{C}$ to $9{ }^{\circ} \mathrm{C}$. The increase from $-1{ }^{\circ} \mathrm{C}$ to $2{ }^{\circ} \mathrm{C}$ might be explained by thermo-rheological processes, observed during testing of bitumen by means of modulated differential scanning calorimetry [15].

Additionally, the variation of (i) the relaxation time of the Kelvin-Voigt unit $\tau_{K V}$, with $\tau_{K V}=\eta / \mu_{v}$ [s], and (ii) the relaxation/creep time $\tau_{3 P}$, with $\tau_{3 P}=\eta /\left(E_{0} / 3+\mu_{v}\right)[\mathrm{s}]$, involving all three parameters of the $3 \mathrm{P}$ model, with the temperature is depicted in Fig. 12(d,e). Whereas $\tau_{K V}$ remains approximately constant from $-4.5^{\circ} \mathrm{C}$ to $2^{\circ} \mathrm{C}$ and increases from $2^{\circ} \mathrm{C}$ to $9^{\circ} \mathrm{C}, \tau_{3 P}$ decreases continuously with increasing temperature. This decrease of $\tau_{3 P}$ reflects the decreasing elastic behavior of bitumen with increasing temperature. 
(a)

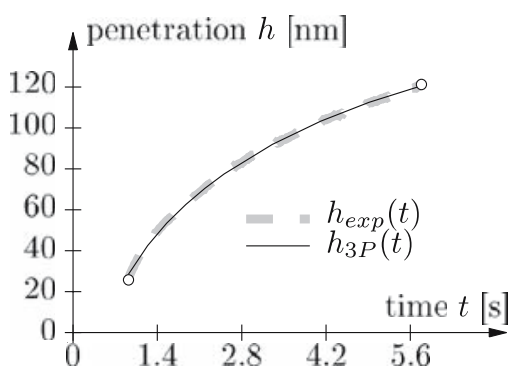

(c) $\mu_{0}[\mathrm{GPa}]$

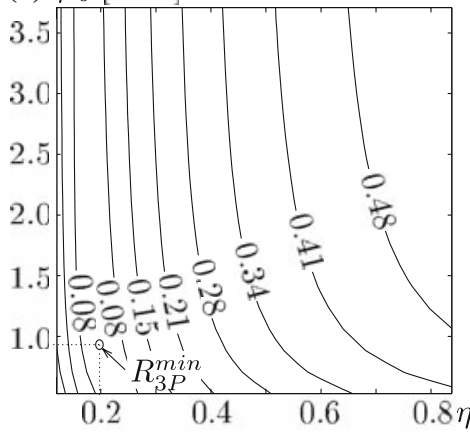

(b) $\mu_{0}[\mathrm{GPa}]$

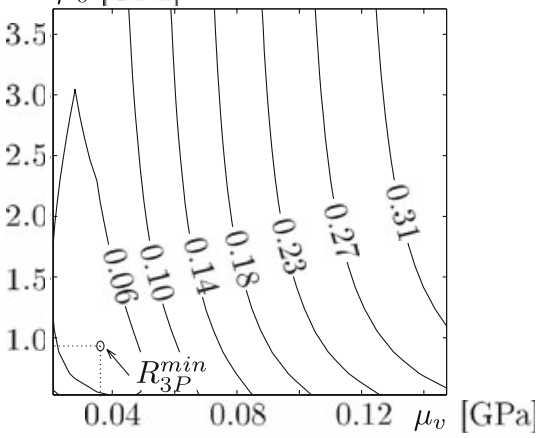

(d) $\eta[\mathrm{GPa} \cdot \mathrm{s}]$

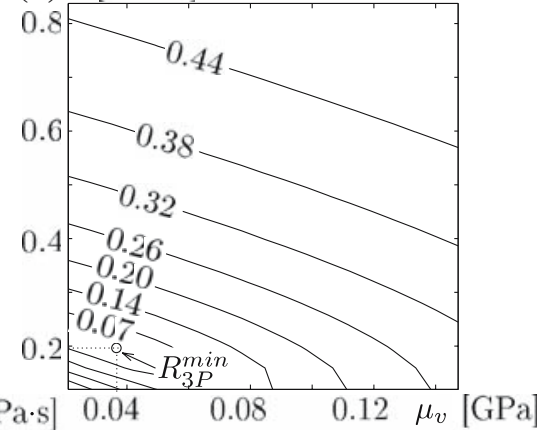

Fig.9 Influence of model parameters on error $R_{3 P}$ : (a) time-penetration curve (NI-test conditions: $T=-1^{\circ} \mathrm{C}, P_{\max }=10 \mu \mathrm{N}$, $\dot{P}=\mathrm{d} P / \mathrm{d} t=40 \mu \mathrm{N} / \mathrm{s}, \tau_{H}=5 \mathrm{~s}$ ), and error $R_{3 P}$ within the parameter range $0.120<\eta<0.838,0.0210<\mu_{\nu}<0.147,0.530$ $<\mu_{0}<3.71$ keeping (b) $\eta=0.199$, (c) $\mu_{v}=0.0351$, and (d) $\mu_{0}=0.883$ constant
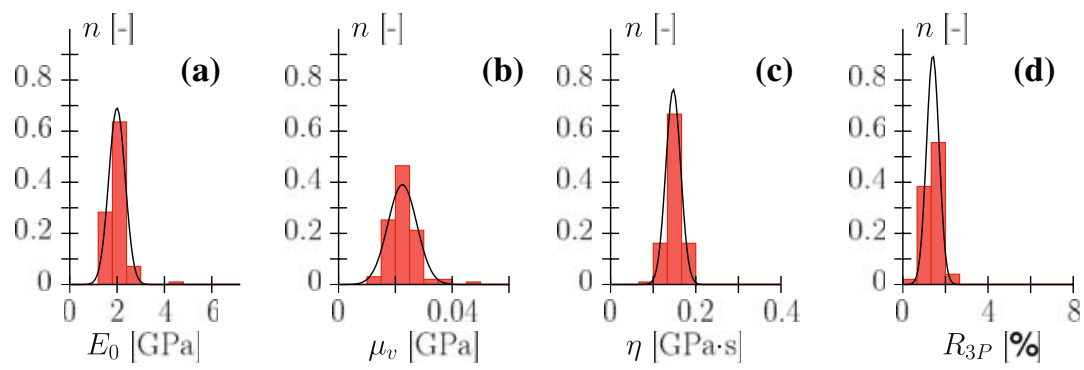

Fig. 10 Identification of model parameters for B50/70 tested at $-1{ }^{\circ} \mathrm{C}$ : frequency plots of (a) Young's modulus of spring in series $E_{0}=3 \mu_{0}$ [GPa], (b) shear modulus of spring in parallel $\mu_{\nu}$ [GPa], (c) viscosity of the dash-pot $\eta$ [GPa.s], and (d) error $R_{3 P}$ [\%] (NI-test conditions: $P_{\max }=20 \mu \mathrm{N}, \dot{P}=\mathrm{d} P / \mathrm{d} t=40 \mu \mathrm{N} / \mathrm{s}, \tau_{H}=5 \mathrm{~s}$ )

The temperature dependence of $\eta$ depicted in Fig. 13 is well-described by an Arrhenius-type law at temperatures above $2^{\circ} \mathrm{C}$, reading:

$\frac{1}{\eta}=\frac{1}{\bar{\eta}(\bar{T})} \exp \left[-\frac{E_{a}}{R}\left(\frac{1}{T}-\frac{1}{\bar{T}}\right)\right]$,

where $\bar{T}[\mathrm{~K}]$ is the reference temperature, $R=8.31$ $\mathrm{J} / \mathrm{MOL} / \mathrm{K}$ is the gas constant, and $E_{\mathrm{a}}[\mathrm{J} / \mathrm{MOL}]$ is the activation energy. The ratio of the activation energy and the gas constant, $E_{\mathrm{a}} / R$, for temperatures above $2^{\circ} \mathrm{C}$ is obtained as $14,600 \mathrm{~K}$. Interestingly, the activation energy computed for the whole tested temperature range (dashed line in Fig. 13), with $E_{\mathrm{a}} / R=9,700 \mathrm{~K}$, agrees very well with the value obtained from macroscopic bending-beamrheometer (BBR) tests of bitumen, revealing a value of $E_{\mathrm{a}} / R$ of $9,000 \mathrm{~K}$ [17]. 

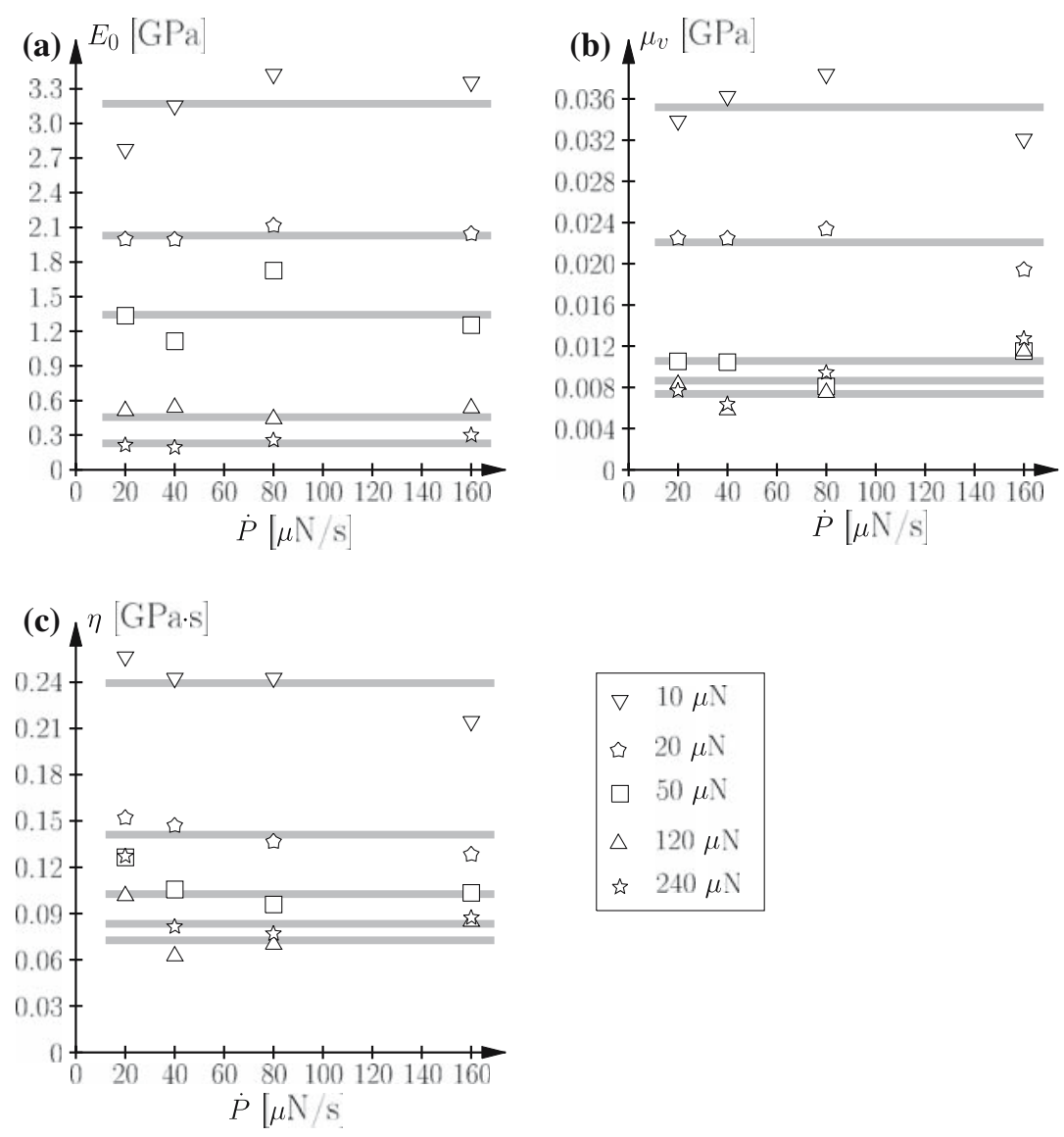

Fig. 11 Influence of loading rate on 3P-model parameters: (a) Young's modulus of spring in series $E_{0}$ [GPa], (b) shear modulus of spring in parallel $\mu_{v}[\mathrm{GPa}],(\mathbf{c})$ viscosity of the dash-pot $\eta\left[\mathrm{GPa}\right.$ ] $\left(\right.$ bitumen B50/70 tested at $T=-1^{\circ} \mathrm{C} ; P_{\max }=10$, $20,50,120,240 \mu \mathrm{N} ; \dot{P}=\mathrm{d} P / \mathrm{d} t=20,40,80,160 \mu \mathrm{N} / \mathrm{s} ; \tau_{H}=5 \mathrm{~s}\left(\right.$ for $\left.P_{\max }=10,20 \mu \mathrm{N}\right)$ and $10 \mathrm{~s}\left(\right.$ for $\left.\left.P_{\max }=50,120,240 \mu \mathrm{N}\right)\right)$

\section{Concluding remarks}

The identification of viscoelastic properties from nanoindentation-test (NI-test) data taking the real tip geometry into account was presented in this paper. Hereby, the shape of the real tip was approximated by an axisymmetric indenter, with the constants $C_{0}$ and $C_{1}$ describing the tip shape. Based on the elastic solution of the indentation problem for this indenter shape [1], the corresponding viscoelastic solution was determined for three deviatoric creep models: (i) the single dash-pot, (ii) the Maxwell, and (iii) the three-parameter model. With the penetration histories for the different viscoelastic models at hand, a parameter-identification procedure for the model parameters was presented.

The application of this procedure was illustrated by determination of the viscoelastic properties of bitumen. For this purpose, NI tests were performed for different loading rates, maximum loads, and at different temperatures, and the respective model parameters were identified. Based on the so-obtained parameters, the following conclusions can be drawn:

1. An increase of the maximum load resulted in decreasing values for the identified model parameters. This effect was explained by the heterogenity of the bitumen microstructure, consisting of both high-viscous strings embedded into a low-viscous matrix.

2. In contrast to the maximum load, affecting the bitumen microstructure to a different extent, the loading rate-which affects the time-dependent, viscoelastic model response and, therefore, reflects the performance of 

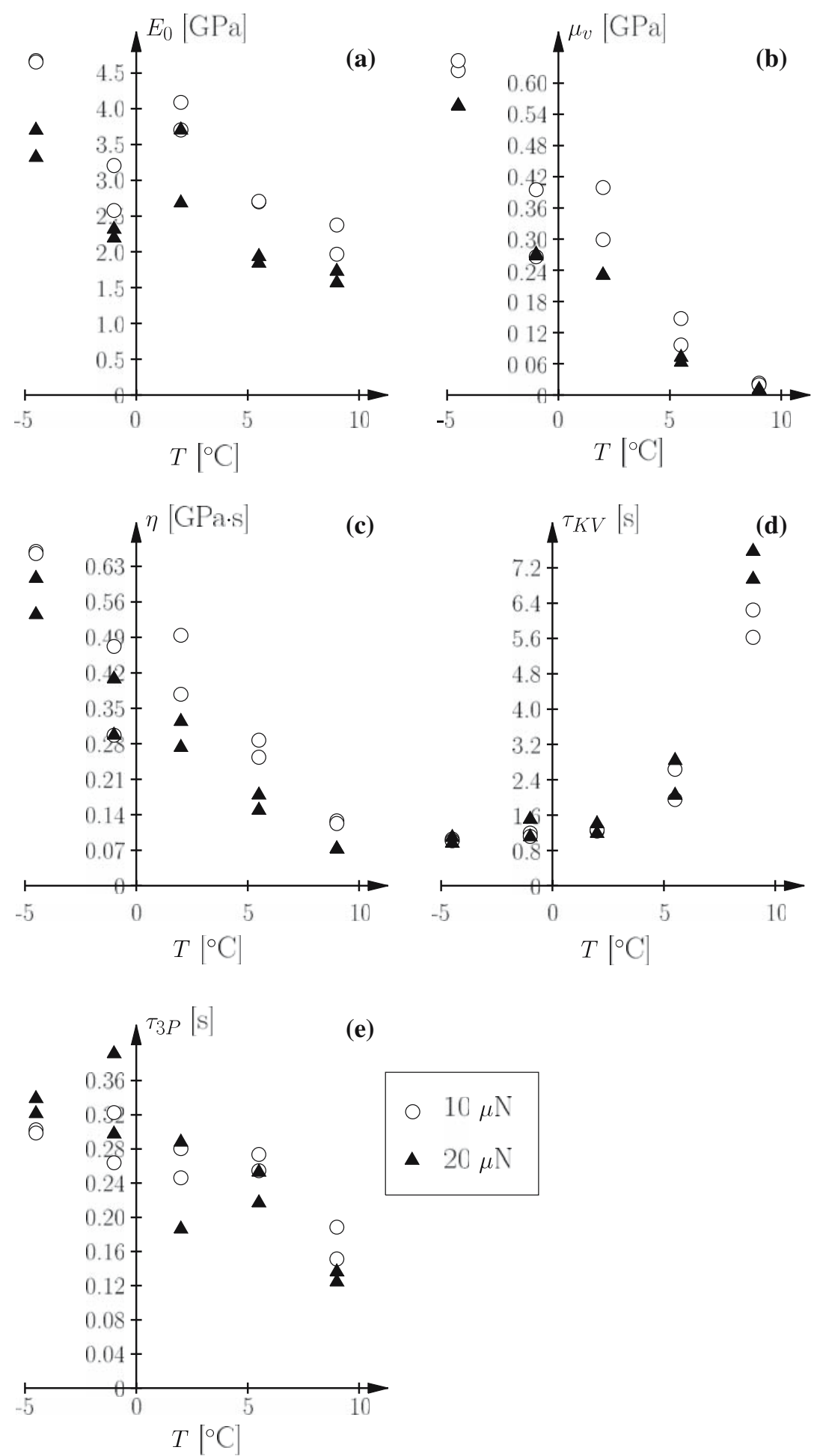

Fig. 12 Temperature dependence of 3P-model parameters: (a) $E_{0}[\mathrm{GPa}]$, (b) $\mu_{v}[\mathrm{GPa}]$, (c) $\eta[\mathrm{GPa} \cdot \mathrm{s}]$, (d) relaxation time of Kelvin-Voigt unit, $\tau_{K V}$ [s], and (e) relaxation/creep time $\tau_{3 P}$ [s] involving all three parameters of the 3P model (NI-test conditions: $\left.P_{\max }=10,20 \mu \mathrm{N}, \dot{P}=\mathrm{d} P / \mathrm{dt}=20,40 \mu \mathrm{N} / \mathrm{s}, \tau_{H}=10 \mathrm{~s}\right)$ 


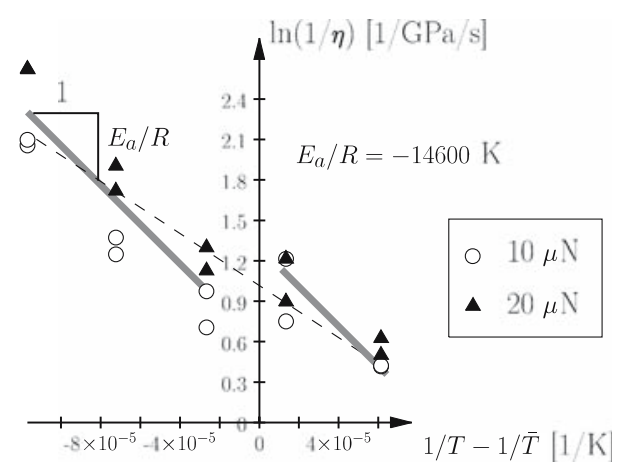

Fig. 13 Identification of Arrhenius-type law describing the temperature dependence of $\eta[\mathrm{GPas}](\bar{T}=273 \mathrm{~K})$

the employed parameter-identification toolshowed no influence on the identified parameters.

3. The temperature dependence of the viscosity $\eta$ was successfully described by an Arrheniustype law.

The three-parameter model employed for parameter identification gave excellent agreement between the experimentally obtained NI-penetration curves and the model response. This agreement in the short-term creep response of bitumen was also observed in the course of BBR tests. However, as reported in [17], for an extended time range of testing, the nonlinear dash-pot is able to capture both the short- and long-term response of bitumen [17]. The extension of the presented parameter-identification approach to more complex visco-elastic models, including the nonlinear dash-pot model, is a topic of ongoing research.

Acknowledgements The authors thank the remaining members of the Christian Doppler laboratory for 'Performance-Based Optimization of Flexible Pavements' at Vienna University of Technology, especially Klaus Stangl for helpful comments and fruitful discussions on the presented research work. Financial support by the Christian Doppler Gesellschaft (Vienna, Austria) is gratefully acknowledged.

\section{References}

1. Sneddon IN (1965) The relation between load and penetration in the axisymmetric Boussinesq problem for a punch of arbitrary profile. Int J Eng Sci 3:47-57
2. Oliver WC, Pharr GM (1992) An improved technique for determining hardness and elastic modulus using load and displacement sensing indentation experiments. J Mater Res 7(6):1564-1583

3. Cheng L, Xia X, Scriven LE, Gerberich WW (2005) Spherical-tip indentation of viscoelastic material. Mech Mater 37:213-226

4. Vandamme M, Ulm F-J (2006) Viscoelastic solutions for conical indentation. Int J Solids Struct 43(10):31423165

5. Lee EH, Radok JRM (1960) The contact problem for viscoelastic bodies. J Appl Mech 82:438-444

6. Abramowitz M, Stegun IA (1972) Handbook of mathematical functions, with formulas, graphs, and mathematical tables. Dover, New York

7. Lee EH (1955) Stress analysis in visco-elastic bodies. Quarter Appl Math 13:183-190

8. Findley WN, Lai JS, Onaran K (1989) Creep and relaxation of nonlinear viscoelastic materials. Dover Publications, New York

9. Partal P (1999) Rheological characterisation of synthetic binders and unmodified bitumens. Fuel 78:1-10

10. ÖNORM EN 1426 (2000) Bitumen und bitumenhaltige Bindemittel - Bestimmmung der Nadelpenetration [Bitumen and bituminous binders - Determination of needle penetration]. Österreichisches Normungsinstitut, Vienna In German

11. ÖNORM EN 12593 (2000) Bitumen und bitumenhaltige Bindemittel - Bestimmmung des Brechpunktes nach Fraaß [Bitumen and bituminous binders - Determination of the Fraass breaking point]. Österreichisches Normungsinstitut, Vienna. In German

12. ÖNORM EN 1427 (2000) Bitumen und bitumenhaltige Bindemittel - Bestimmmung des Erweichungspunktes - Ring- und Kugel-Verfahren [Bitumen and bituminous binders - Determination of softening point - Ring and Ball method]. Österreichisches Normungsinstitut, Vienna. In German

13. Ulm F-J, Delafargue A, Constantinides G (2005) Experimental microporomechanics. In Ulm F-J, Dormieux L (eds) Applied micromechanics of porous materials (CISM Courses and Lectures No. 480), Vienna, Springer

14. Press WH, Teukolsky SA, Vetterling WT, Flannery BP (1996) Numerical recipes in Fortran 77: the art of scientific computing, vol 1 of Fortran numerical recipes. Cambridge University Press, Cambridge

15. Jäger A (2004) Microstructural identification of bitumen by means of atomic force microscopy (AFM), modulated differential scanning calorimetry (MDSC), and reflected light microscopy (RLM). Master's thesis, Vienna University of Technology, Vienna

16. Stangl K, Jäger A, Lackner R (2006) Microstructurebased identification of bitumen performance. Int $\mathrm{J}$ Road Mater Pavement 7:111-142

17. Lackner R, Spiegl M, Blab R, Eberhardsteiner J (2005) Is low-temperature creep of asphalt mastic independent of filler shape and mineralogy? Arguments from multiscale analysis. J Mater Civil Eng (ASCE) 17(5):485-491 